\title{
¿Cómo influye en los pacientes crónicos de hemodiálisis la organización de actividades de ocio durante sus sesiones?
}

\author{
"Premio al mejor trabajo de investigación otorgado por el colegio oficial de enfermería de \\ Sevilla 2011"
}

\section{Maria Guenoun Sanz - MªAntonia Bauça Capellà}

\section{Enfermeras}

Unidad de diálisis. Hosp. Son Espases. Palma de Mallorca

\section{Sr. Director:}

La búsqueda de la satisfacción del paciente crónico en hemodiálisis y la mejora de su calidad de vida es un objetivo estudiado en los últimos años. Los trabajos que abordan esta perspectiva se han multiplicado ante la evidencia de que los pacientes en este tratamiento están sometidos a múltiples estresores físicos y psicosociales que requieren la activación de una serie de estrategias de afrontamiento, no sólo para sobrellevar las demandas y los cambios en el estilo de vida causados por la enfermedad, sino también para hacer frente a sus efectos psicológicos, tales como la incertidumbre, el miedo y la ausencia de control ${ }^{1}$.

Se observa que, en general, la enfermera está mejor preparada para los aspectos técnicos del tratamiento y los parámetros fisiológicos del paciente que para el estado mental del mismo. A veces se actúa cuando la sintomatología es evidente y no se ayuda a prevenirla.

Correspondencia:

$\mathrm{M}^{\mathrm{a}}$ Antonia Bauça Capellà

Centro Sanitario de Balears a Son Espases

C/. Andrea Doria No 55

07014 Palma de Mallorca. Baleares

E-mail: mabc750@hotmail.com
Los profesionales de la salud tienden a asumir que la valoración que realizan del estado de salud y de la Calidad de Vida de los pacientes a su cargo es congruente con la de los propios pacientes. Sin embargo, diversos estudios indican que existen discrepancias. Los aspectos subjetivos como el dolor, la ansiedad y la depresión es donde hay menos concordancia entre la enfermera y el paciente ${ }^{2}$.

Hay que tener en cuenta la diversidad de pacientes en una unidad de hemodiálisis. Se diferencian unos y otros según distintos parámetros importantes que se debe tener en cuenta a la hora de abordar los niveles de salud mental y que, al mismo tiempo, influirán en las estrategias que el paciente utilizará para mejorar su situación: antigüedad, enfermedades concomitantes o pluripatología, edad, sexo, opción a trasplante renal, acceso vascular, horas de duración de la sesión, mayor nivel de estudios, haber desarrollado una actividad laboral más cualificada, tener más limitaciones para realizar las actividades laborales, más dolor o malestar, o más ansiedad o depresión disminuye la satisfacción de una forma estadísticamente significativa ${ }^{5}$. La Calidad de Vida es peor en los mayores de 65 años y portadores de catéter temporal ${ }^{6}$.

Los pacientes que Ilevan menos tiempo en diálisis movilizan la búsqueda apoyo social. También recurren más a la distracción procurando no pensar demasiado en su problema. Así, recurrir a la compañía de otros para distraerse o realizar actividades que resulten 
placenteras tiene efectos positivos porque ayuda a centrar la atención en otros aspectos disminuyendo las emociones negativas y pensamientos repetitivos (rumiación). Por el contrario parece que los que llevan más tiempo tienden al aislamiento social y a la confrontación-oposición, es decir, tratan de evitar estar con otros y reconocen que manifiestan más su enfado por su situación. El estrés mantenido en el tiempo junto con la inhibición emocional y el enojo produce baja respuesta inmune y aumento de la depresión. Contrariamente a otros pacientes crónicos, las personas en diálisis con el tiempo manifiestan empeoramiento en el bienestar psicológico³.

Someterse a la hemodiálisis cuatro horas por día, tres veces por semana, puede resultar ser una experiencia cargada de tedio, además de causar incomodidad durante su realización. Es común que los pacientes relaten que el tiempo parece arrastrarse o durar más. Un estudio brasileño con terapia musical quiso conocer, entre otros objetivos, la percepción del tiempo sensorial de pacientes adultos sometidos a la hemodiálisis, partiendo de que la literatura sobre esta temática es escasa. La conclusión fue que más del $80 \%$ de los pacientes sintió el tiempo pasar más rápido después de las intervenciones musicales ${ }^{8}$.

La necesidad de Virginia Henderson "participar en actividades recreativas" significa practicar actitudes encaminadas a la diversión que comprende la capacidad de disfrutar, de poseer sentimientos positivos sobre uno mismo y sobre los demás, y de sentir placer. Si queremos mantener un adecuado nivel de salud mental, estas actividades no pueden ni deben abandonarse totalmente ${ }^{7}$. La enfermería nefrológica y el resto del equipo de la unidad tienen que abordar al paciente con sospecha de patología ansiosa o depresiva mediante la escucha y practicando todas las terapias de ayuda y apoyo ${ }^{10}$. El clima interpersonal de la unidad de crónicos de diálisis influye en el bienestar del paciente. Contrariamente a las visiones tradicionales, la comunicación abierta en las relaciones de los pacientes con el personal no es perjudicial y contribuye al bienestar?

Todo ello precisa de una intervención enfermera desde otro abordaje que ayude al paciente a mejorar la percepción de su tratamiento crónico. Aliviar el tedio y la monotonía que suponen las sesiones de hemodiálisis es una manera rápida y efectiva de contribuir a cambiar el estado de ánimo de los pacientes al menos durante la duración de las actividades de ocio propuestas por enfermería. Con este estudio se ha querido hacer las sesiones de diálisis menos incómodas, aburridas y más divertidas y socializadoras.

\section{Objetivo}

Cubrir la necesidad de actividades recreativas en el entorno hospitalario.

\section{Metodología}

El estudio emplea la metodología cualitativa descriptiva de investigación participativa.

El campo del estudio fue el turno de tarde de los martes, jueves y sábados de la unidad de hemodiálisis de nuestro hospital. La muestra la constituyó la totalidad de pacientes (15) del turno de tarde y los profesionales de las unidades de crónicos (7) del mismo turno (zonas con serología negativa y positiva).

Las entrevistas fueron realizadas en la sala de hemodiálisis, desde las butacas y camas de cada paciente, obteniendo de ellos los permisos para las mismas.

Los datos se han recogido mediante observación, conversaciones informales y entrevistas semiestructuradas con preguntas abiertas, elaboradas mediante un guión flexible. Las entrevistas fueron grabadas y las grabaciones fueron transcritas al pie de la letra por los entrevistadores.

Se siguieron los siguientes pasos analíticos descritos por Miles y Huberman:

1) Darle códigos a los apuntes de las entrevistas y transcripciones. 2) Anotar las reflexiones y otros comentarios en los márgenes. 3) Identificar fases similares, relaciones entre las variables, patrones, diferencias marcadas entre grupos y secuencias comunes. 4) Elaboración de un conjunto de generalizaciones y sacar conclusiones.

La intervención consistió en la organización de talleres de diferentes actividades recreativas, previo consentimiento de la dirección del hospital: 
1) Se organizó durante dos días una fiesta de disfraces debido a la proximidad del carnaval. Durante esta fiesta, ser realizó un desfile de disfraces para entrar en la unidad, antes de la conexión, con música de carnaval. Tras la conexión de los pacientes, se procedió a escoger, mediante votación, la reina y el rey del carnaval 2011. A los ganadores se les entregó un marco con la foto ampliada. A los demás, una foto de ellos mismos disfrazados como premio de consolación. Se grabaron las entrevistas que recogían las impresiones de pacientes y profesionales antes, durante y después del evento. Una semana después, se habilitó una sala de reuniones para visualizar un cd-resumen. El equipo profesional y los pacientes visualizaron conjuntamente este vídeo compartiendo risas y anécdotas.

2) Taller de mandalas: se descargó por internet e imprimió estos dibujos geométricos para colorear. Se acordó con los pacientes un día concreto para que trajeran lápices de colores. Se proporcionó más lápices para pacientes que no pudieron comprarlos o traerlos. Se hicieron fotos del momento y se recogieron las opiniones de los involucrados en un cuaderno de campo.

Se pidió a los pacientes y profesionales que quisieran participar que firmaran un consentimiento informado para mantener la confidencialidad de los datos recogidos en todos los soportes y poder utilizarlos en un trabajo científico.

\section{Resultados y discusión}

De los 15 pacientes que forman la totalidad de este turno, 5 participaron, con los disfraces, el primer día y 9 el segundo día pudiendo haber sido 11 , pero dos de los pacientes estaban ingresados. De los profesionales, 6 participaron el primer día y 7 el segundo.

10 pacientes pintaron mandalas y 3 profesionales hicieron lo mismo. El segundo día, 6 pacientes quisieron colorear, 1 prefirió escuchar el fútbol y 5 pidieron uno para llevarse a casa y hacerlo el fin de semana. 7 profesionales se sumaron al taller. Se tuvo que cambiar los lapiceros por rotuladores debido a la incomodidad y peor calidad del color de los mismos.

Tras el análisis de los datos se han creado 7 categorías:
1) Percepción del tiempo en la hemodiálisis, 2) necesidad del paciente de actividades recreativas y de ocio, 3) estado de ánimo, 4) relación con los compañeros de tratamiento, 5) implicación de la familia, 6) relación con el equipo profesional, 7) opinión del equipo profesional.

\section{1) PERCEPCIÓN DEL TIEMPO EN LA HEMODIÁ- LISIS}

El tiempo, para los pacientes conectados a la máquina, es un factor importante que influye negativamente en la percepción de la enfermedad y en cómo la encaran. Acudir a las sesiones resulta monótono, pesado y rutinario. Es decir, hay un sentimiento generalizado de que organizar actividades durante las sesiones es positivo. Ha sido sorprendente descubrir las ganas de la mayoría de participar en diferentes propuestas observando el entusiasmo y el seguimiento de las mismas. "Uno no sabe ya qué hacer". "La última media hora de diálisis es mortal". "Queremos ideas que nos evadan de la diálisis". "Y mejor hacerlo durante la diálisis que fuera, al ser más fácil". "Ha sido una grata sorpresa colorear mandalas, me he relajado y he disfrutado". "He dejado de pintar el mandala y he mirado el reloj; se me había pasado una hora volando".

\section{2) NECESIDAD DEL PACIENTE DE ACTIVIDADES RECREATIVAS Y DE OCIO}

Los pacientes opinan que estas actividades son positivas para su bienestar psicológico siendo una importante ayuda en muchos aspectos. La idea ha sido bien acogida y los resultados claramente positivos. Ha habido ilusión por parte de unos pacientes tanto en los días previos a los disfraces como en el día previo a la emisión del vídeo. Acudieron todos al "estreno" y agradecieron con abrazos a la enfermera organizadora de "la sesión de cine" creada para ellos. Todos quieren una copia del cd. "Me he sentido más joven y mejor, me he olvidado de mis problemas, parecía una cría, he pasado gusto cuando la gente me miraba disfrazada en el ascensor". "Es la primera vez que me disfrazo y me ha gustado". "Me he sentido muy bien y he soltado mucha adrenalina". "Yo quiero ser agradable con la gente y hacerla reír y eso es lo más importante que se puede conseguir". "Me he sentido como un chaval de 18 años". "La próxima vez será mejor porque la gente está súper animada". "Me pienso disfrazar el año que viene de lo que siempre he soñado. Ahorraré todo el año para ello". "Voy a venir disfrazada todo el día. Me haré yo el disfraz". "Me ha 
gustado venir disfrazado y el próximo día repetiré". "Querría una copia del vídeo para tenerlo como un recuerdo entrañable de los compañeros". Uno de los pacientes, con el habla y la movilidad afectada debido a un ACV, dijo en su casa la única palabra claramente entendible, "disfraz", para que sus familiares le consiguieran accesorios para el segundo día de carnaval. Tal fue su obstinación que su familiar nos llamó esa tarde para preguntar si celebrábamos algo especial. La siguiente sesión de diálisis posterior a la del carnaval, el paciente se levantó sólo de la silla de ruedas a su cama, algo impensable anteriormente. $Y$ a la semana y media, ya caminaba por el pasillo ayudado por el técnico de transporte sanitario.

\section{3) ESTADO DE ÁNIMO}

Los pacientes saben que hay enfermos más propensos a encerrarse en sí mismos por su delicada situación anímica. Se aíslan del resto durmiendo, demostrando desinterés por su enfermedad o por sus compañeros. Esta iniciativa es, según muchos, una manera de integrar a estas personas tanto voluntariamente como arrastrados por los que sí se atreven. Es interesante ver cómo los pacientes más positivos sienten la necesidad de ayudar a los compañeros que están más desanimados. Algunos de los que no han querido disfrazarse se han reído viendo a los que sí bromeando y charlado con ellos. "La enfermedad es una mala faena". "Yo no me disfrazaré pero me gustará veros a vosotros. Me encanta que os divirtáis pero yo no. Tenéis un humor porque estáis bien. Yo estoy fatal". "lo importante es empezar con todo esto, dar el primer paso". "Servirá para abrirnos mentalmente. Mucha gente se cierra y necesitamos un empujón". "Hay pacientes que no se han disfrazado. Que no se corten y que suelten lo que tienen dentro". "Hay gente bastante desanimada $y$ viendo a la gente de su alrededor, gente contenta, les ayudará". "La gente desanimada, por no quedarse fuera del grupo se sentirán obligados y se animarán". "Ayudará a la gente que no se relaciona con los otros pacientes, que no se abren a los demás, que estén pensando sólo en la máquina que en otra cosa". "Le dejo mi disfraz a quienquiera para que se anime y se lo ponga el segundo día". Una paciente con una situación personal complicada (hermano con esquizofrenia, economía difícil, responsable del cuidado de un progenitor) ha agradecido la realización del taller de mandalas: "Parece que estás en un mundo diferente, te despejas. Una pasada, a mí me encanta. Gracias a la enfermera $X$ lo he descubierto. También los hago en casa cosa que antes no hacía, tiempo perdido, viendo la tele. Me pongo y me olvido. Estoy en blanco y soy feliz. Los nervios interiores se te van. Se me olvidan los problemas, la tensión. Es una cosa interior muy buena para mí. Lo estoy intentando con mi hermano que tiene esquizofrenia a ver si su mente le ayuda. Que no se quite este taller. Me he comprado mi estuche, mis rotuladores, mi carpeta para que no se arruguen. Cuando me ingresen estaré haciéndolos. Las sopas de letras no son lo mismo y te aburren. El dibujar es buscar los colores que te gusten, de mayor a menor, combinarlos, no hay dos mandalas iguales. Me despiertan un sentimiento muy bueno. Además, estaba hoy cabreada (su catéter se colapsaba al inicio de la sesión incluso con la administración de Urokinasa ${ }^{\circledR}$ ), para Ilorar, me he puesto y parece que el catéter ha reaccionado. Desde que he pintado ha dejado de pitar".

\section{4) RELACIÓN CON LOS COMPAÑEROS}

La unidad de hemodiálisis es, para muchos, su segunda casa y los enfermos y equipo sanitario como una familia. El acudir y verse en la unidad y compartir el mismo tratamiento no es suficiente para algunos de ellos. Creen que es positivo introducir a las personas recién llegadas. Muchos de los pacientes quieren establecer un buen clima y quieren introducir a las personas recién llegadas. Realizar una actividad como la propuesta ayuda positivamente a instituir una buena relación interpersonal y a asentar las bases para una futura relación de confianza, amistad y cariño. Muchos coinciden en que estas actividades fomentan y refuerzan la relación con los compañeros estrechando lazos. "La diálisis es nuestra segunda casa y los compañeros y vosotros una familia por el tiempo que pasamos juntos". "Me gustaría hacer cosas juntos". "Hay gente nueva y esto ayudará a romper barreras y nos unirá". "Así estamos todos juntos y todos en armonía". "Esto demuestra que hay un sentimiento hacia los demás compañeros porque no se hace esto por nadie. Se quiere al compañero, es como tener un familiar aquí".

\section{5) IMPLICACIÓN DE LA FAMILIA}

La familia de unos cuantos pacientes se ha involucrado en el disfraz y le ha transmitido apoyo y buen humor. La madre del paciente más joven acudió a la unidad, el primer día de disfraces, con las tres ensaimadas, contenta por la propuesta. También vino a ver la proyección del vídeo riéndose y emocionándose. El padre 
de una de las enfermas iba a venir pero le pudo la timidez, según su hija. Tres pacientes han querido llevar mandalas a sus casas para pintarlos con sus familiares. Tras la entrega de premios, 5 pacientes han colocado su foto en el salón, junto a las demás fotos familiares. Una nieta envió la foto de su abuelo por teléfono a otros miembros de su familia. Un paciente reacio a disfrazarse enseñó su foto a su hija y se rieron. "Mi familia y mi novio me han ayudado a hacer el disfraz". "Mi mujer se reía".

\section{6) RELACIÓN CON EL EQUIPO PROFESIONAL}

Los pacientes valoran la implicación y el esfuerzo del personal de enfermería en su bienestar, sobre todo, mental. Se sienten comprendidos y queridos. Agradecen que se organice este tipo de propuestas y que esté promovido por el personal de enfermería que es el que está más tiempo con ellos y el que les proporciona los cuidados. El clima laboral que les dejamos entrever influye directamente en ellos. El trato con el paciente no debe ser de superioridad sino de comprensión y respeto. Muchos son los profesionales que se involucraron en los días de carnaval. Una enfermera, que se le olvidó su disfraz, llamó a su marido para que le trajera varios atuendos para disfrazarse ella y alguna compañera que quisiera. Otra enfermera vino en su día libre; una paciente se dio cuenta y lo valoró. "Veo que no pasáis de mí". "Me he reído con vosotras al veros disfrazadas y compruebo que sois gente sana y enrollada y eso es importante para nosotros". "Veros a vosotras contentas y con ganas de cooperación es importante para nosotros, disfrutamos con vosotras del día". "Un látigo, en el trabajo, es lo peor que hay". "Somos una gran familia y esto es un apoyo". "Me ha sorprendido la reacción de algunos profesionales, por ejemplo, gente que ha venido en su día libre; Ves que no pasan de ti sino que te apoyan en todo". "Vosotras nos cuidáis todo el año". "Os agradezco que hayáis podido hacer este día realidad".

\section{7) OPINIÓN DEL EQUIPO PROFESIONAL}

La mayoría ha apoyado la idea de forma entusiasta, con su participación activa o con su opinión positiva. Piensan que se debería seguir con más iniciativas de diversión y buen humor porque ayuda a crear un buen ambiente y a mejorar la comunicación con los pacientes. Un nefrólogo opinó que la idea es estupenda porque el paciente verá que se le tiene en cuenta. "Es una manera de participar todos juntos en una actividad de ocio. Ellos verán que somos un poco más iguales". "Tendríamos que hacer más actividades. Hay más relación con los pacientes". "Anima bastante y pasamos un rato divertido, no siempre lo mismo. Viene muy bien en la sala de crónicos. La tarde se hace más amena".

\section{Conclusiones}

El paciente crónico en hemodiálisis posee muchos factores que le pueden inducir a tener una situación de ansiedad o depresión; hay un alto porcentaje de ellos que padecen esta alteración del ánimo. Uno de estos factores es la percepción que tiene el enfermo del tiempo cuando está realizando su tratamiento sustitutivo. Ayudarle a que se divierta, se evada momentáneamente y comparta con sus compañeros ratos agradables y de interacción es una tarea fácil que recae en las manos de los profesionales de enfermería que comparten casi todo el tiempo de la sesión con él. El paciente valora muy positivamente esta implicación enfermera. Se ha comprobado el éxito del taller de mandalas y de los disfraces para carnaval. Los pacientes quieren repetir la experiencia y demandan más actividades. Incluso hacen propuestas de lo que les gustaría.

Hay un gran deseo encubierto de los pacientes por ser partícipes y actores directos de todas aquellas actividades recreativas que puedan realizarse durante las sesiones de hemodiálisis dentro del respeto por el que no quiera participar. Preguntarles por sus gustos y aficiones y por lo que les gustaría hacer significa implicarles en la toma de estas decisiones y conocer su predisposición e inclinación por unas opciones u otras. También ayuda a que valoren la opinión del resto de compañeros, fomenta el debate de unos con otros y el conocimiento del otro. Los pacientes antiguos creen que es una manera de romper el hielo con los pacientes nuevos. Se aprecia un interés real de los que están en mejor predisposición anímica a ayudar a los que menos. Se ha visto una muestra de empatía y preocupación por los más vulnerables.

En cuanto a la relación con el equipo profesional, es básico fomentar un buen clima laboral para que todos nos sintamos a gusto en la unidad. Nuestra satisfacción profesional repercute directamente en el bienestar de los enfermos. Somos un espejo en el que se ven. Gracias a actividades como ésta se fomenta el humor, la risa, la diversión y la relajación de todos dentro del 
respeto mutuo. Simplificar nuestro ejercicio profesional en la mera ejecución de la técnica dialítica empobrece nuestra relación con el paciente y puede desencadenar nuestra desmotivación laboral.

En el futuro, tenemos muchas ideas muy distintas para poner en práctica. Todas ellas acordes con las peticiones de los pacientes, realizables durante la sesión de hemodiálisis y en el espacio que disponemos en la unidad. Son factibles y novedosas para nosotros.

"Está en la imaginación de la enfermera que tenga la iniciativa de organizar las actividades. Y depende de ella que se realicen o no".

\section{Agradecimientos}

Al personal de enfermería del turno de tarde de la unidad de diálisis del hospital universitario Son Espases, Palma de Mallorca.

Recibido: 27 Octubre 2011

Revisado: 30 Noviembre 2011

Modificado: 7 Enero 2012

Aceptado: 10 Febrero 2012

\section{Bibliografía:}

1. R. Jofré, J.M. López Gómez, J. Luño, R. Pérez García, P. Rodríguez Benítez. Tratado de hemodiálisis. Ed. Médica Jims. Segunda edición actualizada. 2006; 39: 808.

2. Covadonga Valdés Arias et.al. Análisis del acuerdo entre la valoración que hacen los pacientes en hemodiálisis de su Calidad de Vida Relacionada con la Salud (CVRS) y la valoración que de ellos hace el personal de enfermería. Rev. Soc. Esp. Enferm. Nefrol 2010; 13 (4): 228,233.

3. B.Ruiz de Alegría Fernández de Retana, Et al. Cambios en las estrategias de afrontamiento en los pacientes de diálisis a lo largo del tiempo. Rev. Soc. Esp. Enferm. Nefrol 2009; 12 (1): 11/17.

4. De los Rios CJL et al. Depresión en pacientes con nefropatía diabética y su importancia en el diagnóstico enfermero. Rev. Mex. Enferm. Cardiológica,2005; 13 (1-2): 9-14.

5. M. Miguel Montoya,C. Valdés Arias,M. Rábano Colino, Y. Artos Montes, P. Cabello Valle,N. de Castro Prieto, A. García León, A. Martínez Villoria, F. Ortega Suárez. Variables asociadas a la satisfacción del paciente en una unidad de hemodiálisis.Rev.Soc. Esp.Enferm.Nefrol 2009;12(1): 19/25.

6. Anna Seguí Gomà, Patricia Amador Peris, Ana Belén Ramos Alcario. Calidad de vida en pacientes con insuficiencia renal crónica en tratamiento con diálisis. www.revistaseden.org. Volumen 13. Número 3. 2010.

7. Mercedes Ugalde Apalategui. Enfermería de salud mental y psiquiátrica. Masson, 2006.

8. Leandro Bechert Caminha, Maria Júlia Paes da Silva, Eliseth Ribeiro Leão. A influência de ritmos musicais sobre a percepção dos estados subjetivos de pacientes adultos em hemodiálise [La influencia de ritmos musicales sobre la percepción de los estados subjetivos de pacientes adultos en hemodiálisis]. Rev Esc Enferm USP -Bra-2009 dic.

9. SWARTZ Richard D.; et al. , Patient-staff interactions and mental health in chronic dialysis patients. Health and Social Work, 33(2), May 2008, pp.8792.

10. E. Moreno Núñez, et.al. Estudio de la prevalencia de trastornos ansiosos y depresivos en pacientes en hemodiálisis. Rev. Soc. Esp. Enferm. Nefrol. 2004; 7 (4): 225/233. 